the forms (10) become

$$
\sum_{i=0}^{n} \sum_{j=c}^{n} \Delta^{(i+j)} f(c) \eta_{i} \eta_{j}
$$

If $\eta_{0}=\eta_{1}=\cdots=\eta_{n-1}=0, \eta_{n}=1$, we see that

$$
\Delta^{(2 n)} f(c) \geqq 0 .
$$

Since $f(x)$ is continuous by hypothesis, we may apply Lemma 2 and deduce that $f(x)$ is analytic in $a<x<b$. In (11) replace $\eta_{i}$ by $\eta_{i} / \delta$ and let $\delta$ approach zero. We thus obtain

$$
\sum_{i=0}^{n} \sum_{j=0}^{n} f^{(i+j)}(c) \eta_{i} \eta_{j} \geqq 0,
$$

and by Lemma 3 , the function $f(x)$ has the form (9). This completes the proof of the theorem.

HARVARD UNIVERSITY

\title{
ARITHMETIC AND IDEAL THEORY OF ABSTRACT MULTIPLICATION*
}

\author{
BY A. H. CLIFFORD
}

If we are given a ring $R$ we may be called upon to answer the following two questions.

1. Is every element of $R$ uniquely decomposable into prime elements?

2. If not can we introduce ideal elements into $R$ such that the resulting system has this property?

Since these questions can be put in terms involving only the operation of multiplication, it is natural to attempt a solution in the same terms. We start, therefore, with a group-like system in which multiplication only is defined, namely a class $S$ satisfying the following postulates:

* A statement of definitions and results of a thesis done under Professors E. T. Bell and Morgan Ward at the California Institute of Technology.

(Added in proof.) I find that ovoid ideals were first discovered by I. Arnold, Ideale in kommutativen Halbgruppen, Recueil Mathématique, Moscou, vol. 36 (1929), pp. 401-407. Arnold proves Theorem 4 for regular ova (which he calls commutative semi-groups), with a slightly different normal ideal arithmetic. 
$P_{1}$. To every pair of elements $a, b$ of $S$ there corresponds an element $c$ of $S$ unique to within equal elements. We write $c=a b$.

$P_{2}$. If $a=a^{\prime}$ and $b=b^{\prime}$, then $a b=a^{\prime} b^{\prime}$.

$P_{3} . a(b c)=(a b) c$ for all $a, b, c$ in $S$.

$P_{4} . a b=b a$ for all $a, b$ in $S$.

$P_{5}$. There exists an element $i$ in $S$ such that $i a=a i=a$ for all $a$ in $S$. This element, evidently unique, is called the identity element of $S$.

Following E. T. Bell, we call such a system an ovum.* An element $a$ of $S$ is said to divide an element $b$ of $S$, written $a \mid b$, if the equation $a x=b$ has a solution $x$ in $S$. Two elements $a, b$ of $S$ are associate, written $a \sim b$, if they divide each other. Elements associate to the identity $i$ are called unities. Nonassociate elements are called essentially distinct. We say that $a$ is a proper divisor of $b$, written $a \| b$, if $a$ divides $b$ but $b$ does not divide $a$. An element of $S$ having a proper divisor in $S$ other than $i$ is called reducible; otherwise, irreducible. An element of $S$ is called decomposable if it is the product of two proper divisors of itself; otherwise, indecomposable. An element of $S$ is called prime if the relation $p \mid a b$ implies that either $p \mid a$ or $p \mid b$, and completely prime if the relation $p^{r} \mid a b$ implies that either $p^{r} \mid a$ or $p^{r} \mid b$, for every positive integer $r$. The index of an element of $S$ is the number of essentially distinct powers thereof. If $a$ is of index $r$, then its first $r$ powers $a, a^{2}, \cdots, a^{r}$ are essentially distinct, and all higher powers are associate to $a^{r}$. If all the powers are essentially distinct, we say that $a$ is of infinite index.

An element $a$ of $S$ is said to be decomposable into irreducible elements if a finite number of essentially distinct irreducibles $p_{1}, p_{2}, \cdots, p_{r}$ and a unit $e$ exist in $S$ such that

$$
a=e p_{1}^{\alpha_{1}} p_{2}^{\alpha_{2}} \cdots p_{r}^{\alpha_{r}}
$$

where the $\alpha_{i}$ are positive integers. The decomposition is said to be unique if the existence of another,

$$
a=e^{\prime} q_{1}^{\beta_{1}} q_{2}^{\beta_{2}} \cdots q_{s}^{\beta_{s}}
$$

* E. T. Bell, Unique decomposition, American Mathematical Monthly, vol. 37 (1930), pp. 400-418. The non-commutative case has been treated by Morgan Ward, Postulates for an abstract arithmetic, Proceedings of the National Academy of Sciences, vol. 14 (1928), pp. 907-911. 
implies that

$$
r=s,
$$

and (by suitable numeration)

$$
p_{i} \sim q_{i}, \quad p_{i}^{\alpha_{i}} \sim q_{i}^{\beta_{i}}, \quad(i=1,2, \cdots, r) .
$$

The second of these is equivalent to the statement that either $\alpha_{i}=\beta_{i}$ or else neither $\alpha_{i}$ nor $\beta_{i}$ is less than the index of $P_{i}$.

An ovum $S$ is said to admit unique decomposition if every element of $S$ is uniquely decomposable into irreducible elements of $S$. The ensuing theorem gives an elementary set of criteria in answer to question 1.

THEOREM 1. The following conditions are necessary and sufficient that an ovum $S$ admit unique decomposition:

I. Teilerkettensatz. If $a_{n+1} \| a_{n}$, then the sequence $a_{1}, a_{2}, \cdots$ must terminate.

II. Every reducible element of $S$ is decomposable.

III. Every irreducible element of $S$ is completely prime.

If $S$ is a ring, II can be replaced $*$ by

IIA. Vielfachenkettensatz. If $a_{n} \| a_{n+1}$, then either the sequence $a_{1}, a_{2}, \cdots$ terminates, or no element $\neq 0$ of $S$ is divisible by every $a_{n}$.

Let us denote by $x A$ the class of elements $x a$ as $a$ ranges over the class $A$. A class $A$ of elements of $S$ is called an ovoid ideal if it includes all elements $s$ of $S$ such that, for every element $x$ of $S, x s$ is divisible by all the common divisors of $x A$ in $S$. This is substantially equivalent to a definition given by $\mathrm{H}$. Prüfer. $\dagger$ If $S$ is a ring, every ovoid ideal is a Dedekind ideal, but the converse is not necessarily true. Ideals will be denoted by small German letters. An ideal is called a principal ideal if it is identical with the set of multiples of a single element of $S$.

A common divisor of a class $A$ of elements of $S$ is called a greatest common divisor (G.C.D.) thereof if it is divisible by all

* Thanks to a clever device invented by A. Fraenkel, Über die Teiler der Null und die Zerlegung von Ringen, Journal für Mathematik, vol. 145 (1915), pp. 139-176.

$\dagger \mathrm{H}$. Prüfer, Untersuchungen ïber Teilbarkeitseigenschaften in Körpern, Journal für Mathematik, vol. 168 (1932), pp. 1-36. 
other common divisors of $A$. It is plainly unique to within associate elements.

THEOREM 2. The condition III of Theorem 1 can be replaced by the condition that every ovoid ideal be a principal ideal. This in turn can be replaced by the two conditions:

IIIA. Every pair $a, b$ of elements of 'S has $a$ G.C.D. $(a, b)$ in $S$. IIIB. $(a, b) c=(a c, b c)$ for all $a, b, c$ in $S$.

By a regular ovum we mean one in which cancellation is permissable, that is, $a c=b c$ always implies $a=b$. Condition II is satisfied for regular ova, and IIIB is a consequence of IIIA, leaving only I and IIIA. These conditions are practically those given by J. Koenig,* so that Theorem 2 is simply an extension of Koenig's elegant result to the irregular case. The sets of criteria (I, IIA, III) and (I, IIA, IIIA, IIIB) for general commutative rings involve only multiplication, yet they are not applicable to unrestricted ova.

Most of the theory of Dedekind ideals can be carried over bodily to that of ovoid ideals. In particular, in the case of regular ova, Krull's form of Noether's conditions $\dagger$ that the set of Dedekind ideals in a ring admit unique decomposition goes over unchanged. For the irregular case we find, as in the proof of Theorem 4 below, that the following set of conditions is useful. This is simply a corollary of Theorem 2 .

THEOREM 3. The following conditions are necessary and sufficient that the set of ovoid ideals of an ovum $S$ admit unique decomposition:

I. Teilerkettensatz: If $\mathfrak{a}_{n+1} \supset \mathfrak{a}_{n}$ (proper inclusion) then the sequence of ideals $\mathfrak{a}_{1}, \mathfrak{a}_{2}, \cdots$ of $S$ must terminate.

II. $\mathfrak{a} \supseteq \mathfrak{c}$ implies the existence of an ideal $\mathfrak{b}$ of $S$ such that $\mathfrak{a} \mathfrak{b}=\mathfrak{c}$.

III. Every reducible ideal is decomposable.

Proceeding now to the second question mentioned at the beginning, we say that an ovum $S$ admits an ovum $\Sigma$ as an ideal arithmetic if $S$ is a subovum of $\Sigma$ and $\Sigma$ admits unique

* J. Koenig, Algebraischen Grössen, 1903, Chapters 1 and 4.

† See B. L. van der Waerden, Moderne Algebra, 1931, vol.2, pp. 97-104. 
decomposition. An ideal arithmetic $\Sigma$ of $S$ is said to be normal if

(i) every element of $\Sigma$ divides some element of $S$;

(ii) if $a \mid b$ relative to $\Sigma, a$ and $b$ being in $S$, then $a \mid b$ relative to $S$;

(iii) every element of $\Sigma$ is the G.C.D. of its multiples in $S$.

THEOREM 4. If an ovum $S$ admits a normal ideal arithmetic $\Sigma$, then the set of ovoid ideals in $S$ also constitutes a normal ideal arithmetic of $S$, being in fact simply isomorphic with $\Sigma$.

This theorem gives only a partial answer to the question, in that it tells us only whether or not a given ovum admits a normal ideal arithmetic of any kind. It does, however, tell us that if an ovum (or ring) admits a normal ideal arithmetic, including Dedekind ideals and Prüfer's "finite" ideal numbers,* then there is essentially only one, which is completely characterized by the three requirements (i), (ii), and (iii). This extends Prüfer's isomorphism between his finite ideal numbers and Dedekind ideals, when these systems admit unique decomposition, to all possible normal ideal arithmetics. The conditions of Theorem 3 are thus criteria for the existence of a normal ideal arithmetic of any kind.

Ovoid ideals bear an important relation to question 1 because of the fact that the condition that every ideal be a principal ideal is necessary if $S$ is to admit unique decomposition. They bear an important relation to question 2 because of the fact that they must admit unique decomposition if $S$ is to admit a normal ideal arithmetic of any kind. The single (extreme) example of the ring of polynomials with integer coefficients shows that Dedekind ideals are far from having either of these properties. Whatever interesting and useful properties other types of ideals may have which ovoid ideals lack, there can be no doubt that, for the specific purpose of answering these two questions, the ovoid variety are in point of fact ideal.

\section{California Institute of Technology}

* H. Prüfer, Neue Begründung der algebraischen Zahlentheorie, Mathematische Annalen, vol. 94 (1925), pp. 198-243. 